\title{
Desempenho e rendimento de carcaça de frangos de corte alimentados com mistura de aditivos fitogênicos na dieta
}

\author{
Natália Thaís Gonçalves Koiyama ${ }^{(1)}$, Alexandre Pires Rosa ${ }^{(2)}$, Marília Terezinha Sangoi Padilha ${ }^{(1)}$, \\ Lenise Schröder Boemo ${ }^{(2)}$, Anelcir Scher(2), Andréia Medianeira da Silva Melo(2) e Mariane de Oliveira Fernandes(2) \\ (1)Universidade Federal de Santa Catarina, Centro de Ciências Agrárias, Campus Itacorubi, Rodovia Admar Gonzaga, no 1.346, \\ CEP 88040-900 Florianópolis, SC, Brasil. E-mail: nataliakoiyama@yahoo.com.br, mariliap@mbox1.ufsc.br (2)Universidade Federal de \\ Santa Maria, Departamento de Zootecnia, Laboratório de Avicultura, Campus Camobi, Avenida Roraima, no1.000, CEP 97105-900 Santa \\ Maria, RS, Brasil. E-mail: alexandreprosa@gmail.com, leniseboemo@hotmail.com, anelcirscher@gmail.com, andreiamelo89@hotmail.com, \\ mariane_of@yahoo.com.br
}

Resumo - O objetivo deste trabalho foi avaliar o efeito de misturas de aditivos fitogênicos na dieta de frangos de corte, sobre seu desempenho zootécnico e rendimento de carcaça. Foram avaliados 660 pintos de sexo misto, entre 1 e 42 dias de idade, em delineamento inteiramente casualizado, com cinco tratamentos e seis repetições de 22 aves (11 machos e 11 fêmeas). Além do controle, foram avaliados tratamentos com antibiótico (10 ppm de virginiamicina) ou com misturas de aditivos fitogênicos: mistura A, 150 ppm de óleos essenciais de alecrim, cravo, gengibre e orégano; mistura $\mathrm{B}, 150 \mathrm{ppm}$ de óleos essenciais de canela, sálvia, tomilho branco e óleo-resina de copaíba; e mistura $\mathrm{A}+\mathrm{B}, 50 \%$ da mistura A e $50 \%$ da B. A mistura B proporcionou maior ganho de peso e melhor conversão alimentar das aves, no período de 36 a 42 dias de idade. No período total do experimento, o antibiótico e a mistura $\mathrm{B}$ proporcionaram maior ganho de peso das aves. $\mathrm{O}$ consumo de ração foi maior com o antibiótico do que com o controle. A mistura $\mathrm{A}+\mathrm{B}$ e o tratamento controle proporcionaram maior rendimento de coxa+sobrecoxa e maior peso relativo do baço. As mistura B e A+B apresentam potencial como melhoradores de desempenho para frangos de corte.

Termos para indexação: Gallus gallus, antibiótico melhorador de desempenho, extratos de plantas, óleos essenciais, óleo-resina de copaíba.

\section{Growth performance and carcass yield of broiler chicken feed diets with blends of phytogenic additives}

\begin{abstract}
The objective of this work was to evaluate the effect of blending phytogenic additives in broiler chicken diets on their growth performance and carcass yield. Six hundred and sixty mixed-sex broiler chicks, from 1 to 42 days of age, were evaluated in a completely randomized design, with five treatments and six replicates of 22 chicks each (11 males and 11 females). Besides the control, treatments with antibiotic (10 ppm virginiamycin) or with blends of phytogenic additives were evaluated: blend A, $150 \mathrm{ppm}$ of a mixture of essential oils from rosemary, clove, ginger, and oregano; blend $\mathrm{B}, 150 \mathrm{ppm}$ of a mixture of essential oils from cinnamon, sage, white thyme, and copaiba oil-resin; and blend $\mathrm{A}+\mathrm{B}$, a mixture of $50 \%$ blend $\mathrm{A}$ and $50 \%$ blend $\mathrm{B}$. Blend $\mathrm{B}$ provided the highest weight gain and better feed conversion rate at the period of 36 to 42 days of age. In the total experimental period, the antibiotic and blend B provided higher broiler weight gain. Feed intake was higher with the antibiotic than with the control. Blend $\mathrm{A}+\mathrm{B}$ and the control treatment provided higher thigh + drumstick yield and higher relative weight of spleen. The blends $\mathrm{B}$ and $\mathrm{A}+\mathrm{B}$ have potential as performance enhancers for broiler chicken.
\end{abstract}

Index terms: Gallus gallus, performance enhancer antibiotic, herbal extracts, essential oils, copaiba oil-resin.

\section{Introdução}

Os antimicrobianos tiveram importante papel no desenvolvimento da avicultura, tendo possibilitado aumentos significativos de produtividade pela sua ação sobre a biota intestinal das aves (Lee et al., 2004). No entanto, o uso constante de antimicrobianos em doses subclínicas tem sido motivo de crescente preocupação, com a possível transmissão e proliferação, ao longo da cadeia alimentar, de bactérias resistentes. Em decorrência disso, vários países baniram a utilização de antibióticos como melhoradores de desempenho na alimentação de animais (Brenes \& Roura, 2010). Dessa forma, a busca por alternativas vem se intensificando, e os produtos extraídos de plantas têm potencial de uso para esse fim. 
Os aditivos fitogênicos são substâncias derivadas de plantas medicinais ou de especiarias, como óleos essenciais e óleo-resinas, que têm efeito positivo sobre a produção e a saúde dos animais (Perić et al., 2009). Eles podem inibir o crescimento microbiano no intestino e melhorar a digestibilidade dos nutrientes, e são reconhecidos como seguros pela agência americana que regula remédios e alimentos (Food and Drug Administration, FDA) (Jang et al., 2007). A propriedade antibacteriana está fundamentada em estudos in vivo ou in vitro, com: sálvia, Salvia officinalis (Delamare et al., 2007); canela, Cinnamomum zeylanicum (López et al., 2007); orégano, Origanum vulgare (Santurio et al., 2007); alecrim, Rosmarinus officinalis (Genena et al., 2008); copaíba, Copaifera officinalis (Santos et al., 2008); tomilho, Thymus vulgaris (Al-Kassie, 2009); gengibre, Zingiber officinale (Malu et al., 2009); e cravo, Caryophyllus aromaticus (Agostini et al., 2012).

Os aditivos fitogênicos podem atuar sobre microrganismos patogênicos encontrados no intestino das aves (Oviedo-Rondón et al., 2006; Jang et al., 2007; Silva et al., 2009) e melhorar o desempenho de frangos de corte (Agostini et al., 2012; Cardoso et al., 2012; Hong et al., 2012). Contudo, os efeitos dessas inclusões na alimentação animal não estão totalmente elucidados e existem poucas pesquisas com aplicações in vivo. Além disso, há possibilidade de ocorrência de sinergismo entre componentes dos produtos fitogênicos (Moleyar \& Narasimham, 1992), o que possibilitaria maior eficácia dos produtos quando usados em misturas (Cseke et al., 2006).

O objetivo deste trabalho foi avaliar o efeito de misturas de aditivos fitogênicos na dieta de frangos de corte, sobre seu desempenho zootécnico e rendimento de carcaça.

\section{Material e Métodos}

O estudo foi realizado em 2011, no Laboratório de Avicultura da Universidade Federal de Santa Maria, RS. Foram utilizados 660 pintos de corte (330 machos e 330 fêmeas) de 1 dia de idade, da linhagem Cobb500, com peso médio de 49,74 $11,18 \mathrm{~g}$. O estudo incluiu a incubação dos ovos e a vacinação dos pintos contra a doença de Marek, de Gumboro e a bouba aviária. As aves foram distribuídas em delineamento inteiramente casualizado, com cinco tratamentos e seis repetições de 22 aves cada uma - metade de cada sexo -, num total de 30 boxes de $2,25 \mathrm{~m}^{2}$, equipados individualmente com bebedouro pendular e comedouro tubular. Como fonte de aquecimento, foi utilizada campânula elétrica com lâmpada de 150 watts na fase inicial.

Os tratamentos consistiram de dieta sem aditivo (controle), dieta com adição de 10 ppm de virginiamicina (antibiótico) e dietas com adição de misturas de aditivos fitogênicos: mistura A, adição de 150 ppm da mistura de óleos essenciais de alecrim (37,5 ppm), cravo (37,5 ppm), gengibre $(37,5 \mathrm{ppm})$ e orégano $(37,5 \mathrm{ppm})$; mistura $\mathrm{B}$, dieta com adição de $150 \mathrm{ppm}$ da mistura de óleos essenciais de canela (37,5 ppm), sálvia (37,5 ppm), tomilho branco (37,5 ppm) e óleo-resina de copaíba (37,5 ppm); e mistura $\mathrm{A}+\mathrm{B}$, dieta com adição de 150 ppm das misturas $\mathrm{A}$ e $\mathrm{B}$, na proporção de $50 \%$ de cada.

Utilizaram-se dietas experimentais fareladas e isonutritivas, formuladas à base de milho e farelo de soja, de acordo com as fases de criação pré-inicial (1-7 dias), inicial (8-21 dias), crescimento (22-35 dias) e terminação (36-42 dias). Foram utilizados os níveis nutricionais padrões do Laboratório de Avicultura (Lavic) para lotes mistos (Tabela 1), adaptados para condições locais, conforme as tabelas brasileiras de aves e suínos (Rostagno et al., 2005). A composição das misturas de aditivos fitogênicos foi realizada por meio da homogeneização dos tipos de óleos, em proporções iguais; e a sua adição à ração foi feita por meio da incorporação prévia em óleo vegetal, que, em seguida, foi adicionado às dietas.

Algumas medidas foram adotadas para submeter as aves a desafios próximos aos que ocorrem em criação comercial: programa de iluminação contínuo (24 horas de luz), cama aviária composta de $50 \%$ de cama reutilizada e bebedouros lavados a cada três dias.

De 1 a 42 dias, a média da temperatura máxima registrada foi de $30,8 \pm 6,4^{\circ} \mathrm{C}$, e a da mínima, de $22,6 \pm 5,0^{\circ} \mathrm{C}$. As aves receberam ração e água à vontade, e a mortalidade foi registrada diariamente durante o período experimental.

As aves foram pesadas aos 7,21, 35 e 42 dias de idade. O ganho de peso, o consumo de ração corrigido pela mortalidade e a conversão alimentar 
corrigida pela mortalidade (consumo:ganho) foram determinados a cada fase de criação. $O$ índice de eficiência produtiva (IEP) foi calculado de acordo com a fórmula IEP = ganho de peso médio diário x (100 - mortalidade)/10 x conversão alimentar.

Três machos de cada unidade experimental, com 42 dias de idade e peso corporal dentro da variação de mais ou menos $2,5 \%$ do peso médio, foram selecionados e identificados. Eles permaneceram em jejum de sólidos de seis a oito horas e foram insensibilizados e abatidos por sangria na veia jugular, conforme os procedimentos de abate humanitário. A depena foi realizada mecanicamente, e a evisceração foi efetuada manualmente, tendo-se removido, também, a cabeça e as patas. O rendimento de carcaça foi calculado de acordo com o peso vivo da ave antes do abate. O rendimento de peito e de

Tabela 1. Composição das dietas $\left(\mathrm{g} \mathrm{kg}^{-1}\right)$ nas diferentes fases de criação dos frangos de corte.

\begin{tabular}{|c|c|c|c|c|}
\hline \multirow[t]{2}{*}{ Ingredientes } & \multicolumn{4}{|c|}{ Idade (dias) } \\
\hline & 1 a 7 & 8 a 21 & 22 a 35 & 36 a 42 \\
\hline Milho & 572,5 & 576,6 & 579,7 & 604,4 \\
\hline Farelo de soja & 358,2 & 347,0 & 335,6 & 313,0 \\
\hline Óleo de soja & 25,0 & 32,0 & 45,0 & 47,9 \\
\hline Fosfato bicálcico & 18,2 & 18,3 & 16,7 & 15,8 \\
\hline Calcário & 10,4 & 9,1 & 8,9 & 8,4 \\
\hline Sal comum & 4,0 & 4,0 & 4,0 & 4,0 \\
\hline Mistura mineral-vitamínica ${ }^{(1)}$ & 5,0 & 5,0 & 5,0 & 5,0 \\
\hline L-lisina & 1,5 & 1,3 & 1,2 & 0,7 \\
\hline DL-metionina & 1,7 & 1,8 & 1,9 & 0,7 \\
\hline L-treonina & 0,0 & 0,1 & 0,3 & 0,1 \\
\hline Inerte $^{(2)}$ & 3,5 & 4,8 & 1,6 & 0,0 \\
\hline \multicolumn{5}{|l|}{ Composição calculada } \\
\hline Energia metabolizável $\left(\mathrm{kcal} \mathrm{kg}^{-1}\right)$ & 3.000 & 3.050 & 3.150 & 3.200 \\
\hline Proteína bruta $\left(\mathrm{g} \mathrm{kg}^{-1}\right)$ & 220,0 & 215,0 & 210,0 & 200,0 \\
\hline Cálcio $\left(\mathrm{g} \mathrm{kg}^{-1}\right)$ & 10,0 & 9,5 & 9,0 & 8,5 \\
\hline Fósforo disponível $\left(\mathrm{g} \mathrm{kg}^{-1}\right)$ & 4,5 & 4,5 & 4,2 & 4,0 \\
\hline Lisina $\left(\mathrm{g} \mathrm{kg}^{-1}\right)$ & 13,6 & 12,5 & 12,1 & 11,0 \\
\hline Aminoácido sulfurado total $\left(\mathrm{g} \mathrm{kg}^{-1}\right)$ & 9,1 & 9,0 & 9,0 & 7,6 \\
\hline Metionina $\left(\mathrm{g} \mathrm{kg}^{-1}\right)$ & 5,7 & 5,7 & 5,7 & 4,4 \\
\hline Treonina $\left(\mathrm{g} \mathrm{kg}^{-1}\right)$ & 8,2 & 8,2 & 8,2 & 7,7 \\
\hline Triptofano $\left(\mathrm{g} \mathrm{kg}^{-1}\right)$ & 2,2 & 2,2 & 2,1 & 1,9 \\
\hline Fenilalanina $\left(\mathrm{g} \mathrm{kg}^{-1}\right)$ & 10,5 & 12,5 & 9,9 & 9,5 \\
\hline Valina $\left(\mathrm{g} \mathrm{kg}^{-1}\right)$ & 10,0 & 0,98 & 9,5 & 9,1 \\
\hline
\end{tabular}

${ }^{(1)}$ Composição por kg do produto: vitamina A, 2.200.000 UI; vitamina D3, 500.000 UI; vitamina E, $5.000 \mathrm{mg}$; vitamina $\mathrm{K} 3,6,60 \mathrm{mg}$; vitamina $\mathrm{B} 1$, $440 \mathrm{mg}$; vitamina B2, $1.150 \mathrm{mg}$; vitamina B6, $926 \mathrm{mg}$; vitamina B12, 3.600 $\mathrm{mg}$; biotina, $36 \mathrm{mg}$; ácido fólico, $250 \mathrm{mg}$; ácido nicotínico, $5.560 \mathrm{mg}$; ácido pantotênico, $3.600 \mathrm{mg}$; Cu, $1.600 \mathrm{mg}$; Fe, 9.998 mg; I, 88 mg; Mg, 11.993 mg; Se, 40 mg; Zn, 10.996 mg; colina, 60.000 mg; metionina, $297.000 \mathrm{mg}$; lisina, $78.000 \mathrm{mg}$; coccidiostático, $12.000 \mathrm{mg} .{ }^{(2)}$ Caulim. coxa + sobrecoxa foi calculado com base no peso da carcaça, assim como o peso relativo do baço, do coração e do fígado.

Os dados foram analisados, para efeito dos tratamentos, pelo procedimento da análise de variância com uso do programa SAS (SAS Institute, Cary, NC, EUA), tendo-se utilizado o seguinte modelo geral: $\mathrm{Y}_{\mathrm{ij}}=\mu+\mathrm{t}_{\mathrm{i}}+\varepsilon_{\mathrm{ij}}$, em que $\mathrm{Y}_{\mathrm{ij}}$ é a variável dependente; $\mu$ é a média geral; $t_{i}$ mede o efeito do tratamento (controle, antibiótico ou mistura de aditivos fitogênicos); e $\varepsilon_{\mathrm{ij}}$ é o erro aleatório residual. As médias foram comparadas pelo teste de Tukey, a $5 \%$ de probabilidade.

\section{Resultados e Discussão}

Aos sete dias de idade, as aves alimentadas com antibiótico apresentaram maior peso corporal que as alimentadas com a mistura $\mathrm{B}$ e a mistura $\mathrm{A}+\mathrm{B}$ (Tabela 2). Aos 21 dias, entretanto, não foram observadas diferenças no peso corporal das aves entre os tratamentos. Aos 35 dias, os tratamentos com antibiótico e mistura A proporcionaram maior peso corporal. Contudo, aos 42 dias, o tratamento mistura A não manteve o bom desempenho. Nessa idade, as aves que receberam antibiótico apresentaram maior peso, mas não diferiram significativamente das aves alimentadas com a mistura B. Hernández et al. (2004) também observaram bom desempenho de tratamentos com extrato de plantas da família Labiatae (sálvia, tomilho e alecrim), aos 35 dias de idade das aves, e, à semelhança do presente trabalho, relataram diminuição da eficácia dos extratos aos 42 dias.

No período de 1 a 21 dias, não houve diferença para as variáveis de desempenho, mas o consumo de ração na fase pré-inicial (1-7 dias) foi menor nas aves alimentadas com a dieta controle e maior nas alimentadas com a dieta com antibiótico. Jang et al. (2007) não verificaram diferença no peso corporal, no consumo de ração, no ganho total e na conversão alimentar entre frangos alimentados com dieta basal ou suplementada com mistura comercial de óleos essenciais (Crina Poultry, ingrediente ativo timol) ou com antibiótico, aos 35 dias. A falta de desafio sanitário pode ter sido a causa para a ausência de diferença, naquele experimento. 
$\mathrm{Na}$ fase de crescimento (22-35 dias), a mistura $\mathrm{A}+\mathrm{B}$ proporcionou menor conversão alimentar que a mistura $\mathrm{B}$, mas não diferiu da conversão alimentar dos demais tratamentos.

Quanto ao ganho de peso, a mistura A possibilitou ganho semelhante ao do tratamento com antibiótico; no entanto, a mistura não manteve esse bom resultado na fase de terminação. Já a mistura $\mathrm{B}$ não proporcionou bom desempenho na fase de crescimento, mas resultou no melhor ganho de peso e na melhor conversão alimentar na fase de terminação (36-42 dias), o que aponta para um ganho compensatório nesta fase, com esse tratamento. A mistura B era composta por óleos essenciais de canela, sálvia, tomilho branco e óleo-resina de copaíba que, além de apresentarem propriedade antibacteriana, podem atuar como estimulante digestivo e anti-inflamatório.
Cardoso et al. (2012) constataram maior ganho de peso e melhor conversão alimentar, dos 36 aos 42 dias de idade, em frangos que receberam suplementação com $60 \mathrm{mg} \mathrm{kg}^{-1}$ de piperina. Porém, no período total de criação ( 8 a 42 dias de idade), os autores relataram que esse efeito não se sobressaiu, contrariamente ao observado com a mistura B no presente trabalho. De acordo com os autores, a administração dessa dose de piperina aumentou as vilosidades do duodeno e do íleo (altura x largura), e resultou em maior superfície de absorção no intestino delgado. Benefícios no desempenho dos frangos com o uso de aditivos fitogênicos têm sido relatados para diversas idades: no período de 14 a 21 e de 28 a 35 dias (Hernández et al., 2004); após a primeira semana de idade (Ciftci et al., 2005); de 29 a 42 dias (Mountzouris et al., 2011); e de 28 a 42 dias (Toghyani et al., 2011). Em comum com

Tabela 2. Peso corporal inicial e final, ganho de peso (GP), consumo de ração (CR), conversão alimentar (CA), mortalidade e índice de eficiência produtiva (IEP) nas fases de criação e no período total de crescimento de frangos de corte alimentados com rações contendo antibiótico e misturas de aditivos fitogênicos ${ }^{(1)}$.

\begin{tabular}{|c|c|c|c|c|c|c|c|}
\hline Variável & Controle & Antibiótico & Mistura A & Mistura B & Mistura A+B & EPM & Valor de $\mathrm{p}$ \\
\hline Peso inicial & 49,23 & 50,14 & 49,67 & 49,73 & 49,39 & 0,11 & 0,0509 \\
\hline & \multicolumn{7}{|c|}{ Pré-inicial (1-7 dias) } \\
\hline Peso final & $172,5 \mathrm{ab}$ & $177,3 \mathrm{a}$ & $175,9 \mathrm{ab}$ & $170,7 b$ & $172,0 \mathrm{~b}$ & 0,82 & 0,0440 \\
\hline GP (g) & 122,8 & 127,2 & 125,9 & 121,0 & 122,8 & 0,78 & 0,0639 \\
\hline $\mathrm{CR}(\mathrm{g})$ & $136,3 b$ & $147,3 \mathrm{a}$ & $144,0 \mathrm{ab}$ & $139,6 \mathrm{ab}$ & $145,0 \mathrm{ab}$ & 1,18 & 0,0138 \\
\hline \multirow[t]{2}{*}{$\mathrm{CA}(\mathrm{g}: \mathrm{g})$} & 1,11 & 1,16 & 1,14 & 1,15 & 1,18 & 0,01 & 0,0955 \\
\hline & \multicolumn{7}{|c|}{ Inicial (8-21 dias) } \\
\hline Peso final & 737,4 & 745,9 & 755,3 & 741,7 & 741,3 & 2,29 & 0,1197 \\
\hline GP (g) & 564,9 & 568,5 & 579,4 & 570,9 & 569,3 & 2,05 & 0,2384 \\
\hline $\mathrm{CR}(\mathrm{g})$ & 900,7 & 942,6 & 896,3 & 942,2 & 929,4 & 7,13 & 0,0837 \\
\hline \multirow[t]{2}{*}{ CA (g:g) } & 1,59 & 1,66 & 1,55 & 1,65 & 1,63 & 0,01 & 0,0655 \\
\hline & \multicolumn{7}{|c|}{ Crescimento (22-35 dias) } \\
\hline Peso final & $1.883,3 b$ & $1.946,1 \mathrm{a}$ & $1.944,1 \mathrm{a}$ & $1.872,9 \mathrm{~b}$ & $1.920,4 \mathrm{ab}$ & 8,28 & 0,0029 \\
\hline GP (g) & $1.145,9 b c$ & $1.200,2 \mathrm{a}$ & $1.188,8 \mathrm{ab}$ & $1.131,2 \mathrm{c}$ & $1.179,0 \mathrm{abc}$ & 7,17 & 0,0031 \\
\hline $\mathrm{CR}(\mathrm{g})$ & $1.778,3$ & $1.847,2$ & $1.830,4$ & $1.810,1$ & $1.795,1$ & 11,23 & 0,3259 \\
\hline \multirow[t]{2}{*}{ CA $(g: g)$} & $1,55 \mathrm{ab}$ & $1,54 \mathrm{ab}$ & $1,54 \mathrm{ab}$ & $1,60 \mathrm{a}$ & $1,52 \mathrm{~b}$ & 0,01 & 0,0266 \\
\hline & \multicolumn{7}{|c|}{ Terminação (36-42 dias) } \\
\hline Peso final & $2.279,4 \mathrm{c}$ & $2.414,3 \mathrm{a}$ & $2.309,0 \mathrm{bc}$ & $2.365,8 \mathrm{ab}$ & $2.350,1 \mathrm{abc}$ & 11,41 & 0,0002 \\
\hline GP (g) & $396,0 \mathrm{ab}$ & $468,2 \mathrm{ab}$ & $364,9 b$ & $492,9 a$ & $429,8 \mathrm{ab}$ & 13,73 & 0,0110 \\
\hline $\mathrm{CR}(\mathrm{g})$ & $1.035,6$ & $1.095,4$ & $1.084,9$ & $1.083,4$ & $1.071,9$ & 7,64 & 0,1083 \\
\hline \multirow[t]{2}{*}{$\mathrm{CA}(\mathrm{g}: \mathrm{g})$} & $2,62 \mathrm{ab}$ & $2,42 \mathrm{ab}$ & $3,08 \mathrm{a}$ & $2,22 \mathrm{~b}$ & $2,51 \mathrm{ab}$ & 0,01 & 0,0206 \\
\hline & \multicolumn{7}{|c|}{ Total (1-42 dias) } \\
\hline GP (g) & $2.229,7 \mathrm{c}$ & $2.364,1 \mathrm{a}$ & $2.259,0 \mathrm{bc}$ & $2.316,1 \mathrm{ab}$ & $2.300,9 \mathrm{abc}$ & 11,40 & 0,0002 \\
\hline $\mathrm{CR}(\mathrm{g})$ & $3.841,0 \mathrm{~b}$ & $4.052,2 \mathrm{a}$ & $3.925,2 \mathrm{ab}$ & $4.005,1 \mathrm{ab}$ & $3.921,7 \mathrm{ab}$ & 22,55 & 0,0197 \\
\hline CA (g:g) & 1,72 & 1,71 & 1,74 & 1,73 & 1,70 & 0,01 & 0,8606 \\
\hline Mortalidade (\%) & 3,79 & 3,03 & 3,79 & 3,03 & 3,79 & 0,74 & 0,9739 \\
\hline IEP $(\%)$ & 296,31 & 319,56 & 298,31 & 309,58 & 309,23 & 3,90 & 0,3303 \\
\hline
\end{tabular}

${ }^{(1)}$ Médias seguidas de letras iguais, nas linhas, não diferem pelo teste Tukey, a 5\% de probabilidade. EPM, erro-padrão da média. 
os resultados do presente trabalho, ressalta-se a importância do efeito da idade nos resultados.

Abildgaard et al. (2010), contudo, não observaram efeitos significativos de óleos essenciais com timol na conversão alimentar.

Ao longo de todo o período experimental (1-42 dias), os frangos alimentados com antibiótico e mistura $\mathrm{B}$ obtiveram maior ganho de peso que os tratamentos controle e mistura $\mathrm{A}$. O consumo de ração também foi maior no tratamento com antibiótico, comparado ao controle. Segundo Hong et al. (2012), o fornecimento de 125 ppm da combinação de óleos essenciais de orégano, anis e frutas cítricas aos frangos de corte, no período de 1 a 42 dias de idade, não apresentou efeito diferente do antibiótico e nem do controle. Os autores destacam a importância da dosagem do óleo essencial, do tipo de dieta basal, de doenças, de fatores de estresse e das condições de alimentação sobre as respostas de desempenho em estudos com óleos essenciais para frangos.

A determinação da dosagem ideal de óleo essencial também é um fator importante. Ertas et al. (2005) verificaram que a dose ideal da combinação de óleos essenciais de óregano, cravo e anis, para frangos, foi de 200 ppm; já Ciftci et al. (2005) relatam a dose de 400 ppm, para óleo essencial de anis, e Agostini et al. (2012), a dose de 100 a 200 ppm, para óleo essencial de cravo.

Ao longo de todo o período experimental, o tratamento controle apresentou menor desempenho zootécnico do que o com antibiótico. Esse resultado é indicativo de que as condições experimentais foram capazes de representar desafio ao bom desenvolvimento dos frangos, o que asseguraria a possibilidade de resposta à inclusão dos aditivos fitogênicos na dieta. Em condições subótimas, a resposta dos animais a esse tipo de tratamento costuma ser mais evidente (Lee et al., 2004).

Os tratamentos não diferiram quanto ao IEP e às taxas de mortalidade.

O peso corporal dos machos abatidos foi maior no tratamento com antibiótico que no controle, na mistura $B$ e na mistura $A+B$ (Tabela 3). Essa diferença, porém, não influenciou o rendimento de carcaça.

A mistura $\mathrm{A}+\mathrm{B}$ proporcionou melhor rendimento de coxa+sobrecoxa, seguida pelo controle (Tabela 3). Isabel \& Santos (2009), apesar de não terem observado diferenças no rendimento de carcaça, relataram que o rendimento de peito foi significativamente maior em frangos que receberam $100 \mathrm{ppm}$ da mistura de cravo e canela, em comparação ao uso de ácidos orgânicos.

Os tratamentos controle e mistura $\mathrm{A}+\mathrm{B}$ resultaram em aumento no peso relativo do baço, comparados à mistura A. O aumento do baço pode decorrer de uma maior mobilização imunológica desencadeada pelos desafios aos quais as aves foram submetidas. Barreto et al. (2008) e Toghyani et al. (2011) não constataram aumento no peso do baço, ao avaliar diferentes aditivos fitogênicos; no entanto, esses autores não submeteram as aves a condições de desafio sanitário.

Os tratamentos não diferiram quanto ao rendimento de carcaça e peito, bem como quanto ao peso relativo do coração e do fígado. Kirkpinar et al. (2010) também não observaram diferenças no rendimento de carcaça e no peso relativo de órgãos, pelo uso de dieta com óleo essencial de orégano e alho e com a mistura de ambos.

Tabela 3. Peso corporal, rendimentos de carcaça e peso relativos de cortes e órgãos de machos abatidos aos 42 dias de idade, alimentados com rações contendo antibiótico e misturas de aditivos fitogênicos ${ }^{(1)}$.

\begin{tabular}{|c|c|c|c|c|c|c|c|}
\hline Variável & Controle & Antibiótico & Mistura A & Mistura B & Mistura $A+B$ & EPM & Valor de $\mathrm{P}$ \\
\hline Peso corporal (g) & $2.564,4 b$ & $2.649,6 \mathrm{a}$ & $2.580,0 \mathrm{ab}$ & $2.532,1 \mathrm{~b}$ & $2.537,3 b$ & 9,08 & $<0,0010$ \\
\hline \multirow[t]{2}{*}{ Rendimento de carcaça (\%) } & 73,13 & 74,04 & 74,46 & 74,08 & 73,11 & 0,20 & 0,1221 \\
\hline & \multicolumn{7}{|c|}{ Peso relativo à carcaça (\%) } \\
\hline Peito & 36,74 & 37,72 & 36,47 & 36,88 & 36,17 & 0,18 & 0,0990 \\
\hline Coxa + sobrecoxa & $28,01 \mathrm{ab}$ & $27,14 \mathrm{c}$ & $27,96 \mathrm{abc}$ & $27,28 b c$ & $28,14 \mathrm{a}$ & 0,13 & 0,0359 \\
\hline Baço & $0,16 \mathrm{a}$ & $0,14 \mathrm{ab}$ & $0,12 b$ & $0,14 \mathrm{ab}$ & $0,16 \mathrm{a}$ & 0,00 & 0,0019 \\
\hline Coração & 0,60 & 0,57 & 0,57 & 0,58 & 0,58 & 0,01 & 0,6808 \\
\hline Fígado & 2,29 & 2,30 & 2,24 & 2,22 & 2,45 & 0,03 & 0,0514 \\
\hline
\end{tabular}

${ }^{(1)}$ Médias seguidas de letras iguais, nas linhas, não diferem pelo teste Tukey, a 5\% de probabilidade. EPM, erro-padrão da média. 


\section{Conclusão}

A mistura, na dieta de frangos de corte, de aditivos fitogênicos à base de óleos essenciais de canela, sálvia, tomilho branco e copaíba, associada ou não à mistura de óleos essenciais de alecrim, cravo, gengibre e orégano, pode proporcionar desempenhos zootécnicos comparáveis ao do uso de antibióticos promotores de crescimento.

\section{Referências}

ABILDGAARD, L.; HOJBERG, O.; SCHRAMM, A.; BALLE, K.M.; ENGBERG, R.M. The effect of feeding a commercial essential oil product on Clostridium perfringens numbers in the intestine of broiler chickens measured by real-time PCR targeting the $\alpha$-toxin-encoding gene $(p l c)$. Animal Feed Science and Technology, v.157, p.181-189, 2010. DOI: 10.1016/j. anifeedsci.2010.03.010.

AGOSTINI, P.S.; SOLÀ-ORIOL, D.; NOFRARÍAS, M.; BARROETA, A.C.; GASA, J.; MANZANILLA, E.G. Role of in-feed clove supplementation on growth performance, intestinal microbiology, and morphology in broiler chicken. Livestock Science, v.147, p.113-118, 2012. DOI: 10.1016/j. livsci.2012.04.010.

AL-KASSIE, G.A.M. Influence of two plant extracts derived from thyme and cinnamon on broiler performance. Pakistan Veterinary Journal, v.29, p.169-173, 2009.

BARRETO, M.S.R.; MENTEN, J.F.M.; RACANICCI, A.M.C.; PEREIRA, P.W.Z.; RIZZO, P.V. Plant extracts used as growth promoters in broilers. Revista Brasileira de Ciência Avícola, v.10, p.109-115, 2008. DOI: 10.1590/S1516-635X2008000200006.

BRENES, A.; ROURA, E. Essential oils in poultry nutrition: main effects and modes of action. Animal Feed Science and Technology, v.158, p.1-4, 2010. DOI: 10.1016/j.anifeedsci.2010.03.007.

CARDOSO, V. da S.; LIMA, C.A.R. de; LIMA, M.E.F. de; DORNELES, L.E.G.; DANELLI, M.G.M. Piperine as a phytogenic additive in broiler diets. Pesquisa Agropecuária Brasileira, v.47, p.489-496, 2012. DOI: 10.1590/S0100-204X2012000400003.

CIFTCI, M.; GÜLER, T.; DALKILIÇ, B.; ERTAS, O.N. The effect of anise oil (Pimpinella anisum L.) on broiler performance. International Journal of Poultry Science, v.4, p.851-855, 2005. DOI: $10.3923 /$ ijps.2005.851.855.

CSEKE, L.J.; KIRAKOSYAN, A.; KAUFMAN, P.B.; WARBER, S.L.; DUKE, J.A.; BRIELMANN, H.L. Natural products from plants. $2^{\text {nd }}$ ed. Boca Raton: CRC Press, 2006. 611p.

DELAMARE, A.P.L.; MOSCHEN-PISTORELLO, I.T.; ARTICO, L.; ATTI-seRAFINI, L.; ECHEVERRIGARAY, S. Antibacterial activity of the essential oils of Salvia officinalis L. and Salvia triloba L. cultivated in South Brazil. Food Chemistry, v.100, p.603-608, 2007. DOI: 10.1016/j.foodchem.2005.09.078.

ERTAS, O.N.; GÜLER, T.; ÇIFTÇI, M.; DALKILIÇ, B.; SIMSEK, Ü.G. The effect of an essential oil mix derived from oregano, clove and anise on broiler performance. International Journal of Poultry Science, v.4, p.879-884, 2005. DOI: 10.3923/ ijps.2005.879.884.

GENENA,A.K.; HENSE, H.; SMÂNIAJUNIOR, A.; SOUZA, S.M. Rosemary (Rosmarinus officinalis) - a study of the composition, antioxidant and antimicrobial activities of extracts obtained with supercritical carbon dioxide. Ciência e Tecnologia de Alimentos, v.28, p.463-469, 2008. DOI: 10.1590/S0101-20612008000200030.

HERNÁNDEZ, F.; MADRID, J.; GARCIA, V.; ORENGO, J.; MEGÍAS, M.D. Influence of two plant extracts on broilers performance, digestibility, and digestive organ size. Poultry Science, v.83, p.169-174, 2004. DOI: 10.1093/ps/83.2.169.

HONG, J.-C.; STEINER, T.; AUFY, A.; LIEN, T.F. Effects of supplemental essential oil on growth performance, lipid metabolites and immunity, intestinal characteristics, microbiota and carcass traits in broilers. Livestock Science, v.144, p.253-262, 2012. DOI: 10.1016/j.livsci.2011.12.008.

ISABEL, B.; SANTOS, Y. Effects of dietary organic acids and essential oils on growth performance and carcass characteristics of broiler chickens. Journal of Applied Poultry Research, v.18, p.472-476, 2009. DOI: 10.3382/japr.2008-00096.

JANG, I.S.; KO, Y.H.; KANG, S.Y.; LEE, C.Y. Effect of a commercial essential oil on growth performance, digestive enzyme activity and intestinal microflora population in broiler chickens. Animal Feed Science and Technology, v.134, p.304-315, 2007. DOI: 10.1016/j.anifeedsci.2006.06.009.

KIRKPINAR, F.; BORA ÜNLÜ, H.; ÖZDEMIR, G. Effects of oregano and garlic essential oils on performance, carcase, organ and blood characteristics and intestinal microflora of broilers. Livestock Science, v.137, p.219-225, 2010. DOI: 10.1016/j. livsci.2010.11.010.

LEE, K.-W.; EVERTS, H.; BEYNEN, A.C. Essential oils in broiler nutrition. International Journal of Poultry Science, v.3, p.738-752, 2004. DOI: 10.3923/ijps.2004.738.752.

LÓPEZ, P.; SÁNCHEZ, C.; BATLLE, R.; NERÍN, C. Vapor-phase activities of cinnamon, thyme, and oregano essential oils and key constituents against foodborne microorganisms. Journal of Agricultural and Food Chemistry, v.55, p.4348-4356, 2007. DOI: $10.1021 /$ jf063295u.

MALU, S.P.; OBOCHI, G.O.; TAWO, E.N.; NYONG, B.E. Antibacterial activity and medicinal properties of ginger (Zingiber officinale). Global Journal of Pure and Applied Sciences, v.15, p.365-368, 2009. DOI: 10.4314/gjpas.v15i3-4.48561.

MOLEYAR, V.; NARASIMHAM, P. Antibacterial activity of essential oil components. International Journal of Food Microbiology, v.16, p.337-342, 1992. DOI: 10.1016/0168-1605(92)90035-2.

MOUNTZOURIS, K.C.; PARASKEVAS, V.; TSIRTSIKOS, P.; PALAMIDI, I.; STEINER, T.; SCHATZMAYR, G.; FEGEROS, $\mathrm{K}$. Assessment of a phytogenic feed additive effect on broiler growth performance, nutrient digestibility and caecal microflora composition. Animal Feed Science and Technology, v.168, p.223-231, 2011. DOI: 10.1016/j.anifeedsci.2011.03.020.

OVIEDO-RONDÓN, E.O.; HUME, M.E.; HERNÁNDEZ, C.; CLEMENTE-HERNÁNDEZ, S. Intestinal microbial ecology of 
broilers vaccinated and challenged with mixed Eimeria species, and supplemented with essential oil blends. Poultry Science, v.85, p.854-860, 2006. DOI: 10.1093/ps/85.5.854.

PERIĆ, L.; ŽIKIĆ, D.; LUKIĆ, M. Application of alternative of growth promoters in broiler production. Biotechnology in Animal Husbandry, v.25, p.387-397, 2009. DOI: 10.2298/BAH0906387P.

ROSTAGNO, H.S.; ALBINO, L.F.T; DONZELE, J.L.; GOMES, P.C.; OLIVEIRA, R.F. de; LOPES, D.C.; FERREIRA, A.S.; BARRETO, S.L.T. Tabelas brasileiras para aves e suínos: composição de alimentos e exigências nutricionais. 2.ed. Viçosa: UFV: DZO, 2005. 186p.

SANTOS, A.O. dos; UEDA-NAKAMURA, T.; DIAS FILHO, B.P.; VEIGA JUNIOR, V.F.; PINTO, A.C.; NAKAMURA, C.V. Antimicrobial activity of Brazilian copaiba oils obtained from different species of the Copaifera genus. Memórias do Instituto Oswaldo Cruz, v.103, p.277-281, 2008. DOI: 10.1590/ S0074-02762008005000015.
SANTURIO, J.M.; SANTURIO, D.F.; POZATTI, P.; MORAES, C.; FRANCHIN, P.R.; ALVES, S.H. Atividade antimicrobiana dos óleos essenciais de orégano, tomilho e canela frente a sorovares de Salmonella enterica de origem avícola. Ciência Rural, v.37, p.803-808, 2007. DOI: 10.1590/S0103-84782007000300031.

SILVA, M.A.; PESSOTTI, B.M.S.; ZANINI, S.F.; COLNAGO, G.L.; RODRIGUES, M.R.A.; NUNES, L.DE.C.; ZANINI, M.S.; MARTINS, I.V.F. Intestinal mucosa structure of broiler chickens infected experimentally with Eimeria tenella and treated with essential oil of oregano. Ciência Rural, v.39, p.1471-1477, 2009. DOI: $10.1590 / \mathrm{S} 0103-84782009005000135$.

TOGHYANI, M.; TOGHYANI, M.; GHEISARI, A.; GHALAMKARI, G.; EGHBALSAIED, S. Evaluation of cinnamon and garlic as antibiotic growth promoter substitutions on performance, immune responses, serum biochemical and haematological parameters in broiler chicks. Livestock Science, v.138, p.167-173, 2011. DOI: 10.1016/j.livsci.2010.12.018.

Recebido em 29 de janeiro de 2014 e aprovado em 28 de fevereiro de 2014 